\title{
Full-Rate, Full-Diversity Adaptive Space Time Block Coding for Transmission over Rayleigh Fading Channels
}

\author{
S. X. Ng, B. L. Yeap and L. Hanzo \\ School of ECS, University of Southampton, SO17 1BJ, UK. \\ Tel: +44-23-8059 3125, Fax: +44-23-8059 4508 \\ Email: $\{$ sxn,bly,lh\}@ecs.soton.ac.uk, http://www-mobile.ecs.soton.ac.uk
}

\begin{abstract}
A full-rate, full-diversity Adaptive Space Time Block Coding (ASTBC) scheme based on Singular Value Decomposition (SVD) is proposed for transmission over Rayleigh fading channels. The ASTBC-SVD scheme advocated is capable of providing both full-rate and full-diversity for any number of transmit antennas, $N_{t}$, provided that the number of receive antennas, $N_{r}$, equals to $N_{t}$. Furthermore, the ASTBC-SVD scheme may achieve an additional coding gain due to its higher product distance with the aid of the block code employed. In conjunction with SVD, the "water-filling" approach can be employed for adaptively distributing the transmitted power to the various antennas transmit according to the channel conditions, in order to further enhance the attainable performance. Since a codeword constituted by $N_{t}$ symbols is transmitted in a single time slot by mapping the $N_{t}$ symbols to the $N_{t}$ transmit antennas in the spatial domain, the attainable performance of the ASTBC-SVD scheme does not degrade, when the channel impulse response values vary from one time slot to the next. Hence, the proposed ASTBC-SVD scheme is attractive in the context of both uncorrelated and correlated Rayleigh fading channels. The performance of the proposed scheme was evaluated, when communicating over uncorrelated Rayleigh fading channels. Explicitly, an $E_{b} / N_{0}$ gain of $2.5 \mathrm{~dB}$ was achieved by the proposed ASTBC-SVD scheme against Alamouti's scheme [1], when employing $N_{t}=N_{r}=2$ in conjunction with 8PSK.
\end{abstract}

\section{INTRODUCTION}

Orthogonal Space Time Block Coding (STBC) [1, 2] constitutes an attractive low complexity technique designed for attaining spatial diversity, when communicating over Rayleigh fading channels. However, no orthogonal STBC schemes exists that are capable of providing both full-diversity ${ }^{1}$ and fullrate transmission ${ }^{2}$ features in the context of systems having more than two transmit antennas communicating using complex modulation constellation [2]. Therefore, a tradeoff has to

\footnotetext{
${ }^{1}$ A system having $N_{t}$ transmit antennas and $N_{r}$ receive antennas is said to have full diversity, when the diversity order is identical to $N_{t} \times N_{r}$.

${ }^{2} \mathrm{~A}$ system is said to have full-rate transmission, when the throughput of the system equals to $\log _{2}(M)$, where $M$ is the number of modulation levels.
}

be found between the code orthogonality and coding rate of STBC schemes employing more than two transmit antennas. Specifically, an orthogonal STBC scheme having a lower coding rate was designed in [2], while a quasi-orthogonal STBC scheme having a full coding rate was proposed in $[3,4]$. Recently, a full-diversity and full-rate transmission orthogonal STBC scheme employing $N_{t}=4$ and QPSK modulation was proposed in [5], where a more complex non-linear processing technique was invoked. Note that all the above-mentioned STBC schemes are capable of attaining only diversity gain, but no coding gain. By contrast, Space Time Trellis Coding (STTC) schemes [6, 7] are capable of achieving both diversity gain and coding gain. However, the complexity of a fulldiversity STTC arrangement grows exponentially with $N_{t}$ [8].

In an effort to reduce the receiver complexity of the mobile unit, substantial research efforts have been devoted to preprocessing the signal at the base station before transmission. These systems include the Maximum Ratio Combining (MRC) transmit scheme $[9,10]$, the Zero-Forcing (ZF) transmit scheme $[11,12]$, the pre-coding scheme of $[13,14]$ and the pre-BLASTDFE system of [15]. These signal preprocessing schemes assume the knowledge of the channel at the base station, which is generated with the aid of channel estimation or by utilising a feedback link from the mobile units. Furthermore, adaptive transceivers [16] also require the knowledge of the channel at the transmitter. Hence, future generations of wireless communication systems are likely to exploit the knowledge of the channel at the transmitter.

In this contribution, we propose an Adaptive STBC (ASTBC) scheme based on Singular Value Decomposition (SVD), where a full-diversity and full-rate performance is always attainable, provided that the number of receive antennas, $N_{r}$, equals to $N_{t}$ and given that the channel is known at the transmitter. The proposed ASTBC-SVD is also capable of attaining a further coding gain with the aid of the appropriate design of the nonbinary block code. Furthermore, the "water-filling" approach [6, 17] was employed for adaptively distributing the transmitted power to the various antennas according to the prevalent channel conditions, in order to further enhance the attainable performance. Note that the SVD was used in [17] to compute the Gaussianinput channel capacity, while here the SVD was used for a different reason, namely for realising a full-diversity STBC ar- 


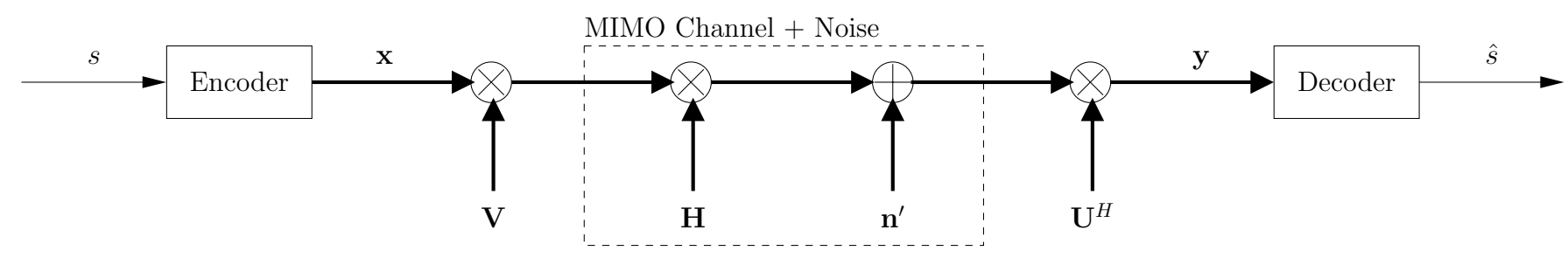

Figure 1: The block diagram of the ASTBC-SVD system.

rangement using a multi-level modulation scheme.

The outline of the paper is as follows. In Section 2 the system's architecture is outlined, while in Section 3 the proposed ASTBC-SVD scheme is evaluated. Finally, we will conclude in Section 4.

\section{SYSTEM OVERVIEW}

With the aid of the SVD [6] any $N_{r} \times N_{t}$ matrix $\mathbf{H}$ may be decomposed as:

$$
\mathbf{H}=\mathbf{U D V}^{H},
$$

where $\mathbf{D}$ is an $N_{r} \times N_{t}$ non-negative and diagonal matrix, while $\mathbf{U}$ and $\mathbf{V}$ are $N_{r} \times N_{r}$ and $N_{t} \times N_{t}$ unitary matrices, respectively. Furthermore, the diagonal entries of $\mathbf{D}$ are the non-negative square roots of the eigenvalues of the matrix $\mathbf{H H}^{H}$. Hence, the Multi-Input Multi-Output (MIMO) channel encountered by a system employing $N_{t}$ transmit antennas and $N_{r}$ receive antennas can be represented by the matrix $\mathbf{H}$. If the transmitter transmits $\mathbf{V x}$, where $\mathbf{x}$ is the $N_{t}$-element transmitted signal vector emanating from the $N_{t}$ antennas, then the $N_{r}$-element received signal vector $\mathbf{y}^{\prime}$ may be expressed using Equation 1 as:

$$
\mathbf{y}^{\prime}=\mathbf{H V} \mathbf{x}+\mathbf{n}^{\prime}=\mathbf{U D x}+\mathbf{n}^{\prime},
$$

where $\mathbf{n}^{\prime}$ is the $N_{r}$-element Additive While Gaussian Noise (AWGN) vector. Note that the multiplication of both sides of Equation 2 by $\mathbf{U}^{H}$ and the multiplication of $x$ by $V$ have only a scaling effect. Hence, we can decouple $\mathbf{y}^{\prime}$ into:

$$
\begin{aligned}
\mathbf{U}^{H} \mathbf{y}^{\prime} & =\mathbf{D} \mathbf{x}+\mathbf{U}^{H} \mathbf{n}^{\prime} \\
\mathbf{y} & =\mathbf{D} \mathbf{x}+\mathbf{n},
\end{aligned}
$$

where we have $\mathbf{y}=\mathbf{U}^{H} \mathbf{y}^{\prime}$ and $\mathbf{n}=\mathbf{U}^{H} \mathbf{n}^{\prime}$. The block diagram of the ASTBC-SVD system is depicted in Figure 1. The number of non-zero eigenvalues of the matrix $\mathbf{H H}^{H}$ is equal to the rank of the matrix $\mathbf{H}$ denoted as $r$, which is given by $r \leq \min \left(N_{t}, N_{r}\right)$. If we have $N_{t}=N_{r}=N$, then $\mathbf{D}$ is a diagonal matrix having $N$ singular values (which are the nonnegative square roots of the eigenvalues of $\mathbf{H H}^{H}$ ) on its diagonal. Hence, the $i$ th element of $\mathbf{y}$, i.e. $y_{i}$ depends only on the $i$ th element of $\mathbf{x}$, i.e. on $x_{i}$ and the $i$ th diagonal element of $\mathbf{D}$, i.e. on $\sqrt{\lambda_{i}}$. In the case of $r<N$ we have $\lambda_{i}=0$ for $i>r$. Hence the ASTBC-SVD scheme of Figure 1 may be

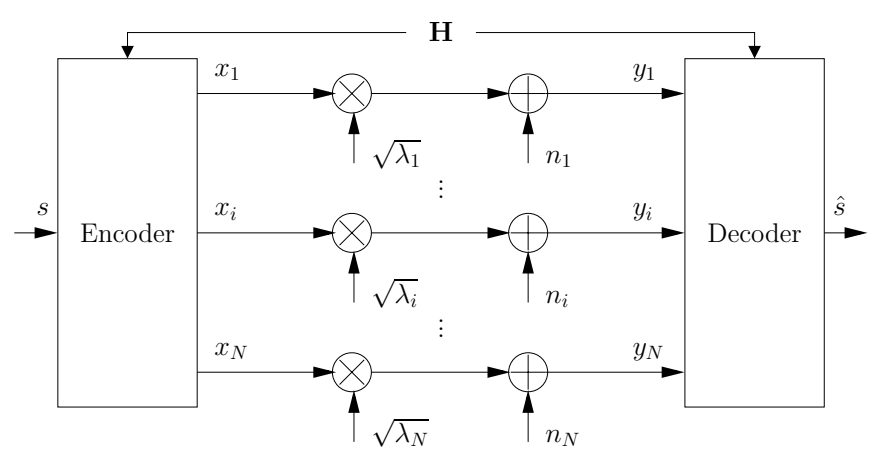

Figure 2: The simplified block diagram of the ASTBC-SVD system.

represented as in Figure 2. Explicitly, the MIMO channel $\mathbf{H}$ is decomposed into $N$ orthogonal subchannels, each having a fading channel power of $\lambda_{i}$, where $i \in\{1, \ldots, N\}$. Therefore, multiplexing gain can be obtained if each element of $\mathbf{x}$ carries independent information, although each subchannel has a different integrity since we have $\lambda_{1} \geq \ldots \geq \lambda_{i} \geq \ldots \geq \lambda_{N}$. However, if the same information symbol is transmitted using $N$ transmit antennas, i.e. if we have identical symbols in the vector $\mathbf{x}$, then we may obtain both transmit and receive diversity, since we have:

$$
\sum_{k=1}^{N} \lambda_{k}=\sum_{i=1}^{N} \sum_{j=1}^{N}\left|h_{i, j}\right|^{2},
$$

where $h_{i, j}$ is the element in the $i$ th row and $j$ th column of the matrix $\mathbf{H}$. However, in order to achieve a full spatial diversity, the channel matrix $\mathbf{H}$ has to be of full rank, i.e. $r=N_{t}=$ $N_{r}=N$. Hence we have to maintain $N_{t}=N_{r}=N$ in order to attain a full spatial diversity of order $N \times N$, although the SVD of a matrix exists for arbitrary matrix dimensions. Furthermore, if an $N$-symbol codeword is transmitted using $N$ transmit antennas, then we may obtain coding gain in addition to the transmit and receive diversity gain.

The block diagram of the ASTBC-SVD encoder is shown in Figure 3. The rate- $1 / N$ Nonbinary Block Code (NBC) of Figure 3 is designed by maximising the minimum product distance $P D_{\min }$ between all possible $N$-symbol codewords. The design rules of this NBC are detailed in $[8,18]$. Note that it is possible to increase the attainable throughput of the proposed ASTBC-SVD scheme by increasing the coding rate of the NBC to $2 / N$ or even to $(N-1) / N$, but we set this de- 


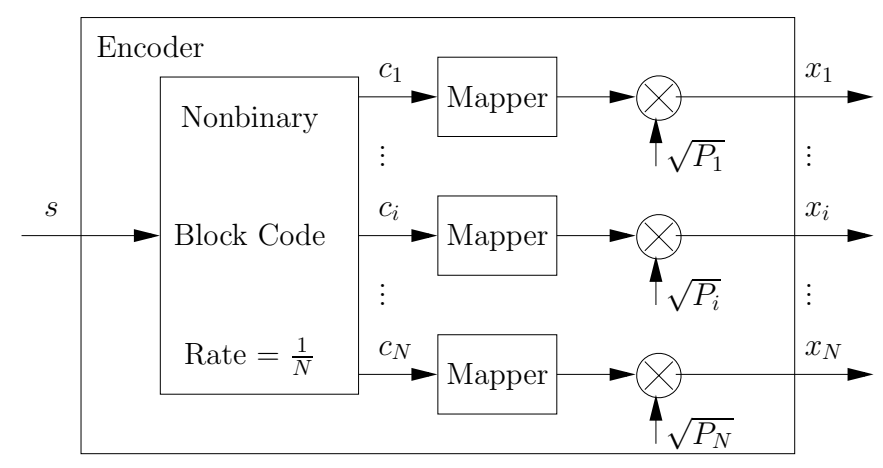

Figure 3: The encoder of ASTBC-SVD.

sign issue aside for future research. The input symbol $s$, where we have $s \in\{0, \ldots, M-1\}$ and $M$ is the number of modulation levels, is encoded by the NBC encoder shown in Figure 3 into the codeword $\mathbf{c}=\left[\begin{array}{lllll}c_{1} & \ldots & c_{i} & \ldots & c_{N}\end{array}\right]^{T}$, where $c_{i} \in\{0, \ldots, M-1\}$ and $i \in\{1, \ldots, N\}$. Then Gray mappers are used for mapping $c_{i}$ to $M$-ary constellation points. Gray-labelling ensures that when a phasor is erroneously detected as the nearest phasor, only a single bit error will occur. By contrast, the natural labelling or Set Partitioning (SP) based labelling may impose more than one bit errors, when a phasor is erroneously detected as the nearest phasor. Hence, upon using Gray-labelling a better Bit Error Ratio (BER) is achieved compared to SP based labelling, despite having the same $P D_{\text {min }}$ and the same symbol error ratio. Next, each phasor is assigned a different transmit power according to the instantaneous channel conditions encountered using the waterfilling principle. Specifically, the channel condition of the ith subchannel is characterised by the $i$ th eigenvalue $\lambda_{i}$ and hence the $i$ th phasor is assigned the transmit power $P_{i}$ according to the water-filling principle [6]:

$$
P_{i}=\left(\mu-\frac{N_{0}}{\lambda_{i}}\right)^{+}, i=1,2, \ldots N,
$$

where $a^{+}$denotes $\max (a, 0), N_{0}$ is the total noise variance and $\mu$ is determined so that we ensure $\sum_{i=1}^{N} P_{i}=P$, where $P$ is the total transmit power. If the transmit power is not assigned adaptively, then we have $P_{i}=P / N$ for all $i$ and we refer to this scheme as the STBC-SVD arrangement. Hence, as it is shown in Figure 3, an information symbol $s$ is first encoded by the rate- $1 / N$ NBC yielding the vector $c$, which is then mapped by the Gray-labelled mappers and multiplied by the $\sqrt{P_{i}}$ factor in order to yield the signal vector $\mathbf{x}=\left[\begin{array}{lllll}x_{1} & \ldots & x_{i} & \ldots & x_{N}\end{array}\right]^{T}$. Hence, power adaptation is carried out during each symbol period for the ASTBC-SVD scheme. Note that if $c_{i}=s$ for all $i$ in Figure 3, we would have an Alamouti-like [1] repetition coded system that does not benefit from additional coding gain. We will refer to the resultant systems as the Space Time Repetition Coding SVD (STRC-SVD) scheme and the Adaptive STRC-SVD (ASTRC-SVD) scheme, respectively.

The detection process is very simple. The received vector $\mathbf{y}^{\prime}$ is first multiplied by $\mathbf{U}^{H}$ for the sake of obtaining $\mathbf{y}$, as in Equation 3. Then the log-domain symbol metrics may be obtained from:

$$
\operatorname{Pr}\left(s=s^{m} \mid \mathbf{y}\right)=-\frac{\sum_{i=1}^{N}\left|y_{i}-\sqrt{\lambda_{i}} x_{i}^{m}\right|^{2}}{N_{0}},
$$

where we have $m \in\{1, \ldots, M\}, M$ is the number of modulation levels, $\mathbf{x}^{m}=\left[\begin{array}{lll}x_{1}^{m} & \ldots & x_{N}^{m}\end{array}\right]$ is a specific codeword related to the $m$ th possible information symbol $s^{m}$ and $N_{0} / 2$ is the noise variance per dimension. These symbol metrics can be passed to an outer channel decoder, if an outer encoder is employed at the transmitter. Otherwise, hard decision is made on these symbol metrics in order to yield the estimate of $s$. Therefore, in our current system the decoder shown in Figure 1 is a simple demodulator based on Equation 6.

\section{PERFORMANCE RESULTS}

In this section we evaluate the attainable performance of the ASTBC-SVD, STBC-SVD, ASTRC-SVD and STRC-SVD schemes using 4PSK, 8PSK and 16PSK, when communicating over uncorrelated Rayleigh fading channels.

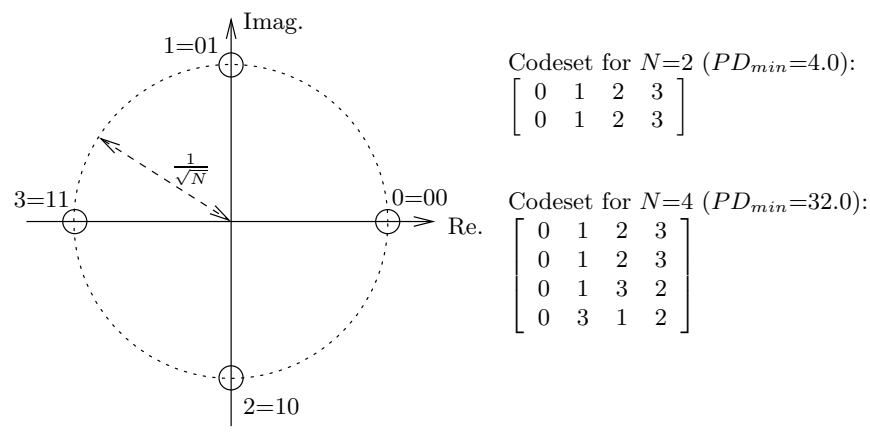

Figure 4: The Gray-labelled 4PSK constellation and the NBC codesets of (A)STBC-SVD for $N=2$ and 4 .

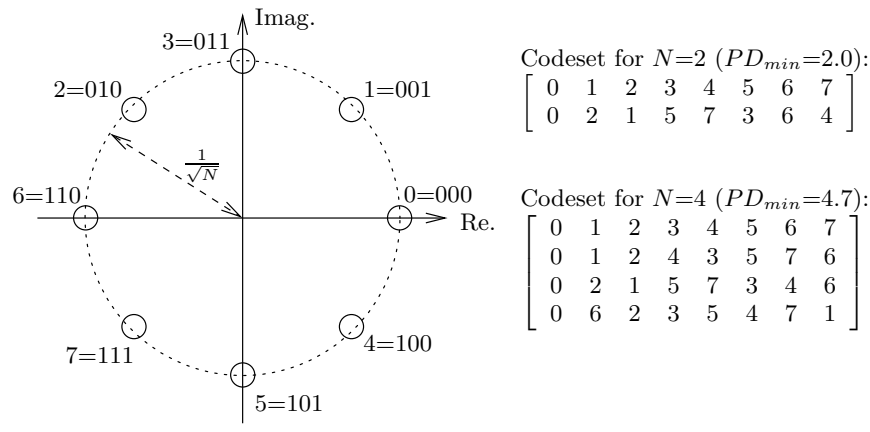

Figure 5: The Gray-labelled 8PSK constellation and the NBC codesets of (A)STBC-SVD for $N=2$ and 4.

The Gray-labelled 4PSK and 8PSK constellations and the NBC codesets of the (A)STBC-SVD scheme designed for $N=$ 2 and 4 are shown in Figures 4 and 5, respectively. Due to the complexity of code design, we only study the NBC codesets 


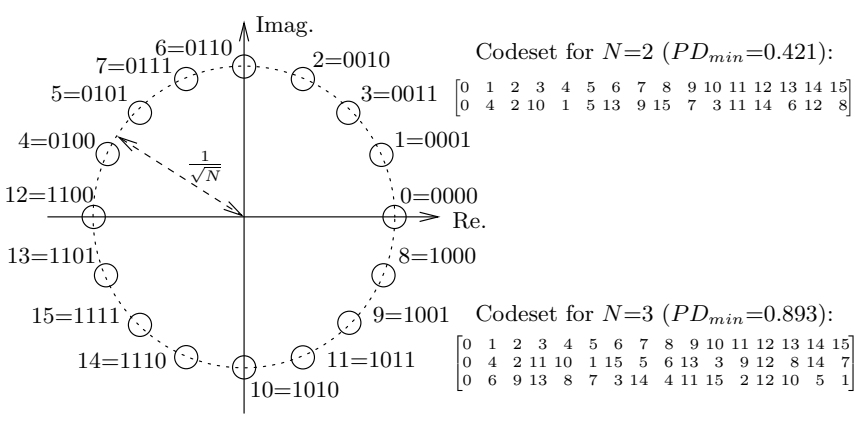

Figure 6: The Gray-labelled 16PSK constellation and the NBC codesets of (A)STBC-SVD for $N=2$ and 3.

for $N=2$ and 3, when the Gray-labelled 16PSK constellation shown in Figure 6 is employed by the (A)STBC-SVD scheme. The minimum product distance $P D_{\min }$ of the code is calculated by assuming that we have $P_{i}=1 / N$ for all $i$. As for the (A)STRC-SVD scheme, which employs repetition codes, each row of the codeset matrix is identical to the first row.

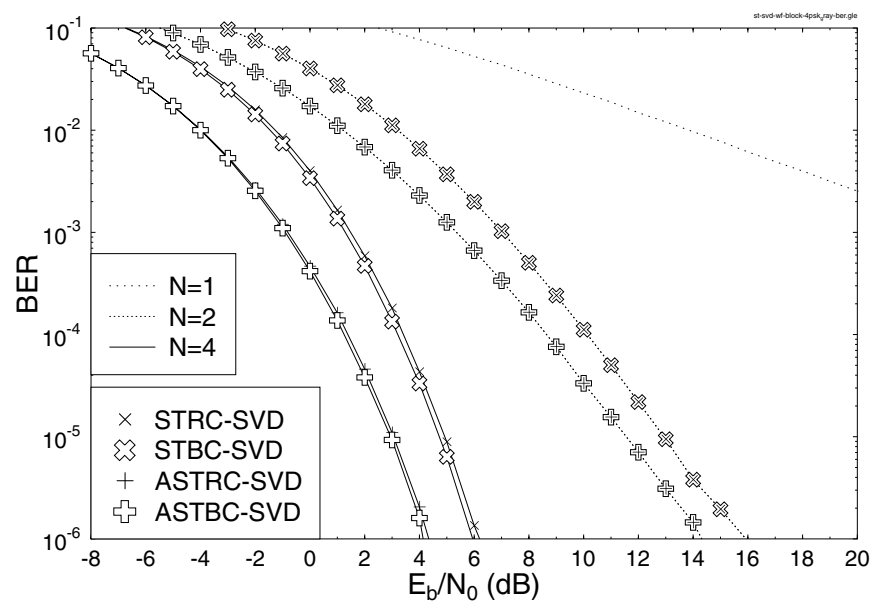

Figure 7: Performance of the ASTBC-SVD, STBC-SVD, ASTRC-SVD and STRC-SVD schemes using 4PSK modulation over uncorrelated Rayleigh fading channels.

Figures 7, 8 and 9 show the achievable performance of the ASTBC-SVD, STBC-SVD, ASTRC-SVD and STRC-SVD schemes, when communicating over uncorrelated Rayleigh fading channels and employing the GRAY-labelled 4PSK, 8PSK and 16PSK constellations, respectively. It is worth mentioning here that the performance of Alamouti's orthogonal STBC scheme utilising $N_{t}=N_{r}=N=2$ is identical to that of the STRC-SVD scheme having $N=2$ and characterised in Figures 7,8 and 9.

As we can see from Figure 7, the performance of the 4PSKbased (A)STBC-SVD and (A)STRC-SVD schemes is identical for $N=2$, since the block code employed by (A)STBC-SVD has an identical $P D_{\min }$ to that of the repetition code employed by (A)STRC-SVD. When $N=4$, the performance of the 4PSK-based (A)STBC-SVD arrangement is only marginally

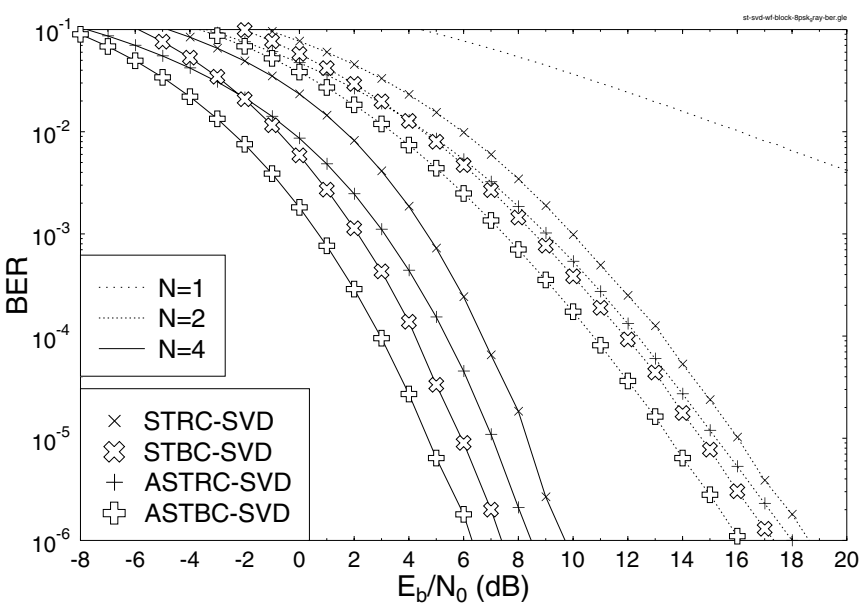

Figure 8: Performance of the ASTBC-SVD, STBC-SVD, ASTRC-SVD and STRC-SVD schemes using 8PSK modulation over uncorrelated Rayleigh fading channels.

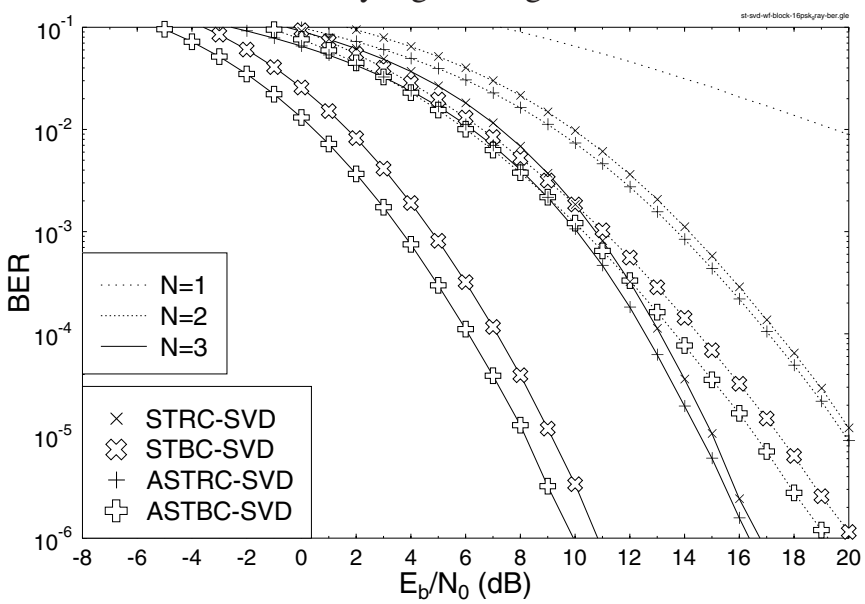

Figure 9: Performance of the ASTBC-SVD, STBC-SVD, ASTRC-SVD and STRC-SVD schemes using 16PSK modulation over uncorrelated Rayleigh fading channels.

better than that of (A)STRC-SVD. However, when the number of modulation levels is higher, the corresponding block code exhibits a higher $P D_{\min }$ compared to that of the repetition code. More specifically, the block coding technique employed by the STBC-SVD arrangement managed to achieve about a $1.2 \mathrm{~dB}$ and $3 \mathrm{~dB}$ coding gain over the STRC-SVD that achieved only diversity gain, when employing $N=2$ as well as 8PSK and 16PSK constellations, respectively. As we can see from Figures 7, 8 and 9, the coding gain of (A)STBC-SVD also increases when $N$ increases. Explicitly, the coding gain of the 16PSK-based ASTBC-SVD scheme increased from $3 \mathrm{~dB}$ to $6.2 \mathrm{~dB}$ compared to ASTRC-SVD, when $N$ increased from 2 to 3 , as shown in Figure 9.

Let us now study the power gain of the water-filling based adaptive technique invoked by the ASTBC-SVD and ASTRCSVD schemes. As we can see from Figures 7, 8 and 9, the per- 
formance of the ASTBC-SVD and ASTRC-SVD schemes is always better than that of their non-adaptive STBC-SVD and STRC-SVD counterparts. More explicitly, the ASTBC-SVD scheme attains an approximately $1.3 \mathrm{~dB}, 1.2 \mathrm{~dB}$ and $0.9 \mathrm{~dB}$ of power gain when compared to their STBC-SVD counterparts in the context of 4PSK, 8PSK and 16PSK constellations, respectively, with the aid of $N=2$ pairs of transmit-receive antennas.

By combining the block coding and adaptive power distribution techniques, the proposed ASTBC-SVD scheme outperformed the STRC-SVD arrangement by $2.5 \mathrm{~dB}$ and $3.5 \mathrm{~dB}$, when employing $N=2$ and 4, respectively, in the context of 8PSK. By contrast, the proposed ASTBC-SVD scheme outperformed the STRC-SVD arrangement by $3.6 \mathrm{~dB}$ and $6.9 \mathrm{~dB}$, when using $N=2$ and 3 , respectively, in the context of 16PSK.

If we can only afford the employment of $N_{r}^{0}$ receive antennas, where we have $N_{r}^{0}<N_{t}$, we can readily partition the transmit antennas into $N_{t} / N_{r}^{0}$ subgroups, where each group has $N_{r}^{0}$ transmit antennas, while transmitting independent information in each transmit antenna group, in order to increase the system's achievable throughput.

\section{CONCLUSION}

We have proposed a simple adaptive STBC scheme that always guarantees achieving both full-diversity and full-rate transmission. If the required channel attenuation knowledge is available at the base station equipped with $N_{t}$ transmit antennas and the mobile can afford using $N_{r}^{0} \leq N_{t}$ receive antennas, we can always attain both a full-rate transmission and a diversity gain of order $N_{r}^{0} \times N_{r}^{0}$ along with both coding gain and power gain. Furthermore, the decoder is simple, incurring a low complexity. Our future research may consider the employment of various high-rate ASTBC-SVDs, soft-decoded channel codecs and high-order modulation schemes.

\section{ACKNOWLEDGEMENTS}

The financial support of the EPSRC, Swindon UK and the EU under the auspices of the PHOENIX, NEWCOM and NEXWAY projects is gratefully acknowledged

\section{REFERENCES}

[1] S. M. Alamouti, "A simple transmitter diversity scheme for wireless communications," IEEE Journal on Selected Areas in Communications, vol. 16, pp. 1451-1458, October 1998.

[2] V. Tarokh, H. Jafarkhani and A.R. Calderbank, "Space-Time Block Codes from Orthogonal Designs," IEEE Transactions on Information Theory, vol. 45, pp. 1456-1467, July 1999.

[3] H. Jafarkhani, "A quasi-orthogonal space-time block code," IEEE Transactions on Communications, vol. COM-49, pp. 1-4, January 2001.

[4] N. Sharma, C. B. Papadias, "Improved quasi-orthogonal codes through constellation rotation," IEEE Transactions on Communications, vol. COM-51, pp. 332-335, March 2003.
[5] L. He and H. Ge, "A New Full-Rate Full-Diversity Orthogonal SpaceTime Block Coding Scheme," IEEE Communications Letters, vol. 7, pp. 590-592, December 2003.

[6] B. Vucetic and J. Yuan, Space-Time Coding. New York: John WileyIEEE Press, May 2003.

[7] V. Tarokh, N. Seshadri and A. R. Calderbank, "Space-time codes for high rate wireless communication: Performance analysis and code construction," IEEE Transactions on Information Theory, vol. 44, pp. 744-765, March 1998.

[8] M. Tao and R. S. Cheng, "Diagonal Block Space-time Code Design for Diversity and Coding Advantage over Flat Rayleigh Fading Channels," IEEE Transactions on Signal Processing, pp. 1012-1020, April 2004.

[9] A. F. Naguib, A. Paulraj and T. Kailath, "Capacity improvement with base-station antenna arrays in cellular CDMA," IEEE Transactions on Vehicular Technology, vol. 43, pp. 691-698, August 1994.

[10] R. L. Choi, K. B. Letaief and R. D. Murch, "MISO CDMA transmission with simplified receiver for wireless communication handsets," IEEE Transactions on Communications, vol. COM-49, pp. 888-898, May 2001.

[11] D. Gerlach and A. Paulraj, "Adaptive transmitting antenna arrays with feedback,” IEEE Signal Processing Letters, vol. 1, pp. 150-152, October 1994.

[12] P. Vandenameele, L. Van Der Perre, M. G. E. Engels, B. Gyselinckx, H. J. De Man, "A combined OFDM/SDMA approach," IEEE Journal on Selected Areas in Communications, vol. 18, pp. 2312-2321, November 2000.

[13] M. Tomlinson, "New automatic equalizer employing modulo arithmetic," IEE Electronics Letters, pp. 138-139, March 1971.

[14] H. Harashima and H. Miyakawa, "Matched-transmission technique for channels with intersymbol interference," IEEE Transactions on Communications, vol. COM-20, pp. 774-780, August 1972.

[15] L. Choi and R. D. Murch, "A pre-BLAST-DFE technique for the downlink of frequency-selective fading MIMO channels," IEEE Transactions on Communications, vol. COM-52, pp. 737-747, May 2004.

[16] L. Hanzo, C. H. Wong and M. S. Yee, Adaptive Wireless Transceivers: Turbo-Coded, Turbo-Equalized and Space-Time Coded TDMA, CDMA and OFDM Systems. New York, USA: John Wiley, IEEE Press, 2002.

[17] E. Telatar, "Capacity of multi-antenna Gaussian channels," European Transactions on Telecommunication, vol. 10, pp. 585-595, Nov-Dec 1999.

[18] S. X. Ng, F. Guo and L. Hanzo, "Iterative Detection of Diagonal Block Space Time Trellis Codes, TCM and Reversible Variable Length Codes for Transmission over Rayleigh Fading Channels," in IEEE Vehicular Technology Conference, (Los Angeles, USA), September 2004. 